\title{
Superior Pedicle Reduction Mammoplasty Supported by Central Glandular Pedicle
}

\author{
MOHAMED A. MEGAHED, M.D.*; YASER M. ELSHEIKH, M.D.*; \\ QUTAIBAH ALKINDARI, FACHARZT** and MOHAMMAD S. ISMAIL, M.Sc.** \\ The Department of Plastic and Reconstructive Surgery, Faculty of Medicine, Menoufia University*, Egypt and \\ Al Babtain Center for Burn, Plastic and Reconstructive Surgery, Ministry of Health**, Kuwait
}

\begin{abstract}
Objectives: To evaluate effect of supporting superior pedicle by central glandular pedicle in reduction mammoplasty in improving aesthetic outcome and reducing the incidence of nipple areola necrosis and postoperative NAC loss of sensation.

Background: Superior pedicle reduction mammoplasty provides good aesthetic outcome, but has high possibility of nipple areola complex loss of sensation, there for keeping the central glandular portion as support to superior pedicle ensures good blood and sensory supply to NAC.

Methods: This study included 37 patients presented by breasts hypertrophy from March 2019 to March 2021. All patients' breasts were examined for suprasternal notch to nipple distance, areolar diameter, nipple to infra mammary fold distance. Postoperative assessment included: Nipple areola complex vascularity, nipple areola complex sensitivity and patients' satisfaction about breast contour, projection, symmetry, scars and overall aesthetic outcome using a score out of 4. Patients underwent marking using wise pattern for skin incision. Intraoperative; excision of skin, fat and glandular tissue inferior to NAC, starting from skin and went directly to chest wall keeping central breast mound with the superior pedicle. Finally closure was in vertical or inverted T pattern according to skin excess to be removed.

Results: In this study we operated upon 37 patients who were asking for breast reduction aged between 20 and 57 years with mean age 39.6 years. The overall complication percentage $14.8 \%$ excluding poor scarring. We had no cases of total Nipple areola complex loss, low incidence of nipple areola complex persistent reduced sensation after 1 year of follow-up (2.7\%) and no complete loss of sensation in any patient. Aesthetic outcome accepted by $97.3 \%$ of patients.

Conclusion: Superior pedicle reduction mammoplasty supported by central glandular pedicle helps in ensuring reliable blood perfusion and sensory nerve supply to nipple areola complex which reduces possibility of post-operative nipple areola complex necrosis and loss of sensation, while patient gets pleasant and long standing aesthetic outcome.
\end{abstract}

Key Words: Superior pedicle - Reduction - Mammoplasty Glandular-Pedicle.

\section{INTRODUCTION}

Many techniques have been described for breast reduction. Recently, plastic surgeons changed the concept of depending on the skin to achieve results needed and have concentrated on depending on structural supportinstead [1].

Most of the well-known used techniques have both an incision pattern and a specific pedicle, sotechnique of breast reduction may be named after the pedicle and skin incision pattern used. For example, the vertical scar type of skin incision pattern may be combined with different pedicles, includingsuperior, inferior, central, lateral or medial pedicle [2].

Lassus, in 1969 described a technique using a superior dermoglandular pedicle for changing position of the nipple areola complex and a vertical scar to close. The shape of the breast was formed by the medial and lateral pillars approximation using skin sutures only. In 1994, Lejour described a modification of Lassus' technique in which liposuction was done before excision to get rid of fat forming part of the breast volume; the skin surrounding the excised area was undermined to help closure of the vertical scar; the superior dermoglandular pedicle was fixed by sutures to the pectoral fascia then, re-approximation of the pillars using sutures in the breast parenchyma to produce a more persistent breast shape. Gathering of the skin of the vertical wound was used to prevent the scar from reaching the infra-mammary crease [3].

Superior pedicle reduction mammoplasty is widely used technique that achieves good aesthetic and long standing result but poor nipple areola complex sensation is a frequent complication with this technique, due to sectioning deep Nipple areola 
complex innervation via Wuringer septum at $5^{\text {th }}$ rib and lateral branch of anterior intercostal and superficial branch of lateral intercostal nerves. Inferior pedicle reduction mammoplasty solved these problems but bottoming out is common drawback [4].

\section{MATERIAL AND METHODS}

This study was carried out after approval of The Ethical Committee of Menoufia Faculty of Medicine and included 37 patients presented by breasts hypertrophy at the Department of Plastic Surgery; Menoufia University Hospital and Al-Babtain Center for Plastic Surgery; Ministry of health; Kuwait from March 2019 to March 2021. Informed consent was obtained from all patients and agreed voluntarily to share and use photos for research with protection of their confidentiality and anonymity. Patients' ages varied from 20 to 57 years with an average of 39.6 years. Patients presented with breasts hypertrophy and were asking for reduction mammoplasty. All patients underwent breasts reduction using superior pedicle reduction mammoplasty supported by central glandular pedicle.

\section{Statistical analysis:}

Data collected entered and analyzed using Microsoft Excel software. Data was then imported into Statistical Package for the Social Science (SPSS version 20.0) software for analysis where quantitative variables were expressed as mean, SD and range and qualitative variables as number and percentage.

Inclusion criteria: Patients presented with Breasts hypertrophy with stable general condition.

Exclusion criteria: Huge pendulous breast, Patients with chronic diseases and cardiac patients, Patients unfit for surgery, Patient with history of breast reduction, Patients who have history of breast cancer, Patient who has unrealistic expectations.

Preoperative preparation: All patients subjected to full history taking, general examination, breasts and axillae examination and breast measurements obtained involving: Suprasternal notch to nipple distance, areolar diameter and nipple to infra mammary fold distance. Photographs were taken preoperatively for all patients.

Preoperative investigations: Routine laboratory investigations, Mammogram for Patients older than 40 years old and ultrasound for those younger than 40 years old, Informed consent was taken including preoperative photography documentation.
Markings: It was done while patients were in standing position, suprasternal notch and midclavicular points were marked, infra mammary lines were marked bilaterally. A line was drown extending from suprasternal notch downward to the umbilicus. Meridian of the breast was marked in each side by a line drown from mid-clavicular point down until crossing infra-mammary fold, dividing the breast to equal halves.

The new nipple areola complex site superior border was located at the level of infra mammary fold on breast meridian line and this could be achieved by inserting one hand behind the breast and the projecting point was the site of the new areola complex superior border. New nipple site wasn't elevated more than $(5 \mathrm{~cm}$ to $7 \mathrm{~cm})$ from the original nipple site.

New areola site marking was drown as an open circle either by using a preformed pliable wire or by free hand (length of the line drown as open circle should be between 13.2 to $14.5 \mathrm{~cm}$ ).

Medial and lateral vertical incision lines that extended from new areola incision line downward were being drown by holding the breast and rotating it superomedially then superolaterally while drawing a line that is in continuity with the meridian of the breast medially and laterally. The two vertical lines were joined together about 2 to $4 \mathrm{~cm}$ higher than Infra mammary fold level. The superior pedicle was marked as the area from the new areola site upper border to below the original areola and between medial and lateral vertical incision lines, keeping a $2.5 \mathrm{~cm}$ border around the areola.

Surgical technique: The procedure was done under general anesthesia while the patient was laying in supine position with both arms abducted 90 degree and fixed to arm support. A prophylactic antibiotic (cefuroxime $1.5 \mathrm{gm}$.) was given intravenously after induction of anesthesia induction.

Following sterilization by $10 \%$ bovidone iodine solution, and toweling, adrenaline/saline 1:200000 was infiltrated at incision sites and de-epithelialization site about 10 to 15 minutes before incision. A tourniquet was used to increase the tension of the skin and to facilitate the de-epithelialization. Nipple areola complex was marked using cookie cutter and de-epithelialization was done at the superior pedicle site and extending to below the nipple areola complex. Incision was done along the marked vertical lines and extended deep under the skin medially and laterally.

The whole breast was pulled up perpendicular to the chest wall using a silk suture through nipple 
areola complex, held up with a clamp by the assistant. The surgeon was holding the lower pole of the breast with a hand and started an en-block excision of the skin, fat and gland as outlined by skin markings using a blade or the diathermy in the other hand.

Excision started below NAC and carried out vertically to the chest wall removing the inferior portion which could be extended laterally and to a lesser extent medially if more excision was needed, preserving superior central glandular portion which ensures preservation of rich blood supply and nerve supply to NAC and provides good projection (Fig. 1), then tissues at inframammary crease was thinned to prevent the development of lateral dog ears.
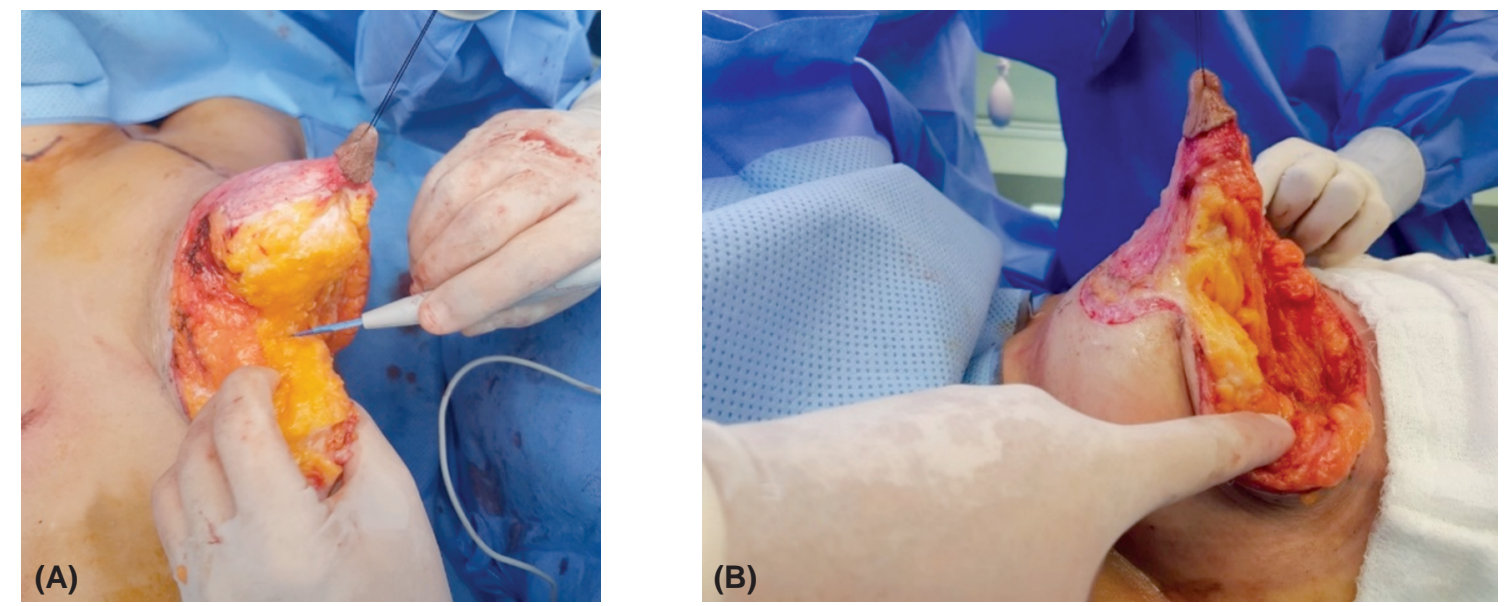

Fig. (1): (A) Lower pole of breast is hold by surgeon's hand and cutting started below areola and extended directly to pectoral fascia. (B) Lateral view showing breasts after excision and the preserved superior central portion.

Breast shaping and closure: Skin incision was extended up to $2 \mathrm{~cm}$ above the superior end of the vertical incision lines to help easy in-setting of nipple areola complex to its new place. Stay sutures were used initially to hold nipple areola complex in its new place. Suction drain size 12 was finally inserted and fixed before closure.

A parenchymal pillar sutures between medial and lateral pillars were used to shape the breast and to help the improving of long term breast projection and prevention of bottoming out. Heavy PDS sutures (size 1) were used, and usually 1 or 2 sutures were used and tightened to the extent which gives the desired shape. Skin staples used temporarily to close the skin to check the shape and presence of dog ears that would need excision.

Final closure were done in 2 layers. Subcutaneous layer were closed using $3 / 0$ monocryl around NAC and 2/0 monocryl in closing vertical and transverse lines. Skin were closed using subcuticular 4/0 monocryl for all wounds.

Dressing were done using Mepelex Border, Advasil or oppsite dressing according to availability. A dressing window was made over NAC to allow for easy inspection and monitor of NAC vascularity. A corset of appropriate size was applied.
Post-operative care: Two doses of cefuroxime $750 \mathrm{mg}$ were given 8 hours apart and 8 hours apart from the induction dose. NAC vascularity was monitored over $1^{\text {st }}$ post-operative day. Wound checked $2^{\text {nd }}$ post-operative day and breasts were examined to rule out hematoma. Drains were removed when they collect less than $30 \mathrm{ml}$.

Patients were instructed to avoid lifting heavy objects or doing vigorous activity for 4 weeks. Remaining suture ends were trimmed about 2 weeks after surgery, and silicon sheet or gel was advised to be used for 3 months to help scars to fade out.

Post-operative evaluation included nipple areola complex vascularity, sensation of the nipple-areola complex (NAC) 2 weeks, 3 months, and 6 months post-operatively. The nipple and four cardinal points of the areola were tested as regard:

- Touch sensation was measured with SemmesWeinstein monofilaments.

- Temperature with hot $\left(40^{\circ} \mathrm{C}\right)$ and cold $\left(4^{\circ} \mathrm{C}\right)$ metal probes.

Aesthetic outcome was evaluated as follow: (A) Patient satisfaction rate was assessed by giving every patient a self-administration questionnaire in which she was asked to rate her satisfaction regarding the following items: Breast contour, 
projection, symmetry, scars, and aesthetic outcome satisfaction. (b) Using a response format ranging from very pleased (score of 4), accepted (score of 3 ), not accepted (score of 2) and very disappointed (score of 1).

Post-operative follow-up was performed as early follow-up for 1 month and the late followup intervals at 3 months, 6 months and 1 year.

\section{RESULTS}

In this study we operated upon 37 patients who were asking for breast reduction aged between 20 and 57 years old with mean age 39.6 in Plastic Surgery Department in Menofia University and Al-Babtain Center for Plastic, Reconstructive and burn surgeries with average resected tissue weight per breast was $561.4 \mathrm{~g}$.

The overall complication percentage was $14.8 \%$ excluding poor scarring and transient nipple areola complex loss of sensation. We had Hematoma in 1 breast $(1.3 \%)$ which needed evacuation in OT. Partial wound dehiscence occurred in 3 breasts (4.1\%) which was managed using dressing then wounds closed by secondary sutures, while no patient got complete wound dehiscence. Partial NAC necrosis happened in 2 breasts $(2.7 \%)$, but there was no complete NAC necrosis. Transient
NAC loss of sensation occurred in 11 breasts which showed improvement over follow-up period except for 2 breasts $(2.7 \%)$ remained with permanent NAC reduced sensation, while there was no cases with complete loss of sensation. Breast fat necrosis happened in 2 breasts (4\%) which was managed conservatively using hot foments and NSAID. Poor scarring occurred in 14 breasts (18.9\%). There were no complications in 39 breasts $(52.7 \%)$. (Table 1$)$.

It was found that $79.7 \%$ of patients didn't loss sensation and $20.3 \%$ of patients had transient loss or reduction of NAC sensation. $2.7 \%$ of patients (1 case) got permanent reduction of NAC sensation, while there was no cases with complete loss of NAC sensation. (Graph 1).

Regarding post-operative aesthetic outcome according to the satisfaction scale is summarized in Table (2). Contour was not accepted by 2 patients $(5.5 \%)$, accepted by 9 patients $(24.3 \%)$ and 26 patients $(70.2 \%)$ were very pleased. Scars were very disappointing for one patient $(2.7 \%)$, was not accepted by 6 patients (16.2\%), accepted by 14 patients $(37.8 \%)$ and 16 patients $(43.2 \%)$ were very pleased. Symmetry was accepted by 13 patients $(35.1 \%)$ and 24 patients $(64.9 \%)$ were very pleased. Aesthetic outcome wasn't accepted by 1 patient $(2.7 \%)$, Accepted by 5 patients $(13.6 \%)$ and 31 patients were very pleased $(83.7 \%)$.
Fig. (2): Front and lateral views of pre-operative and 1 year post-operative follow-up of 55 years old patient.
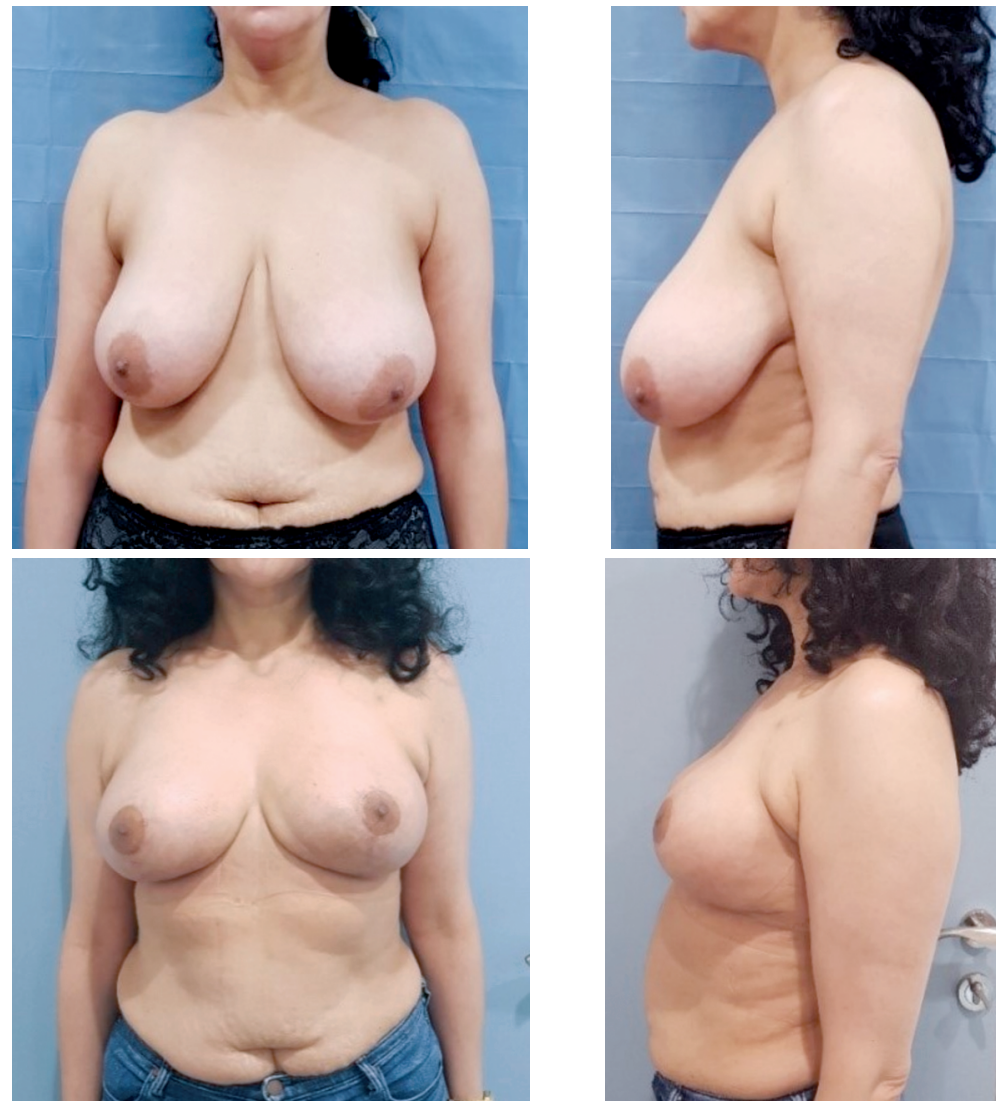

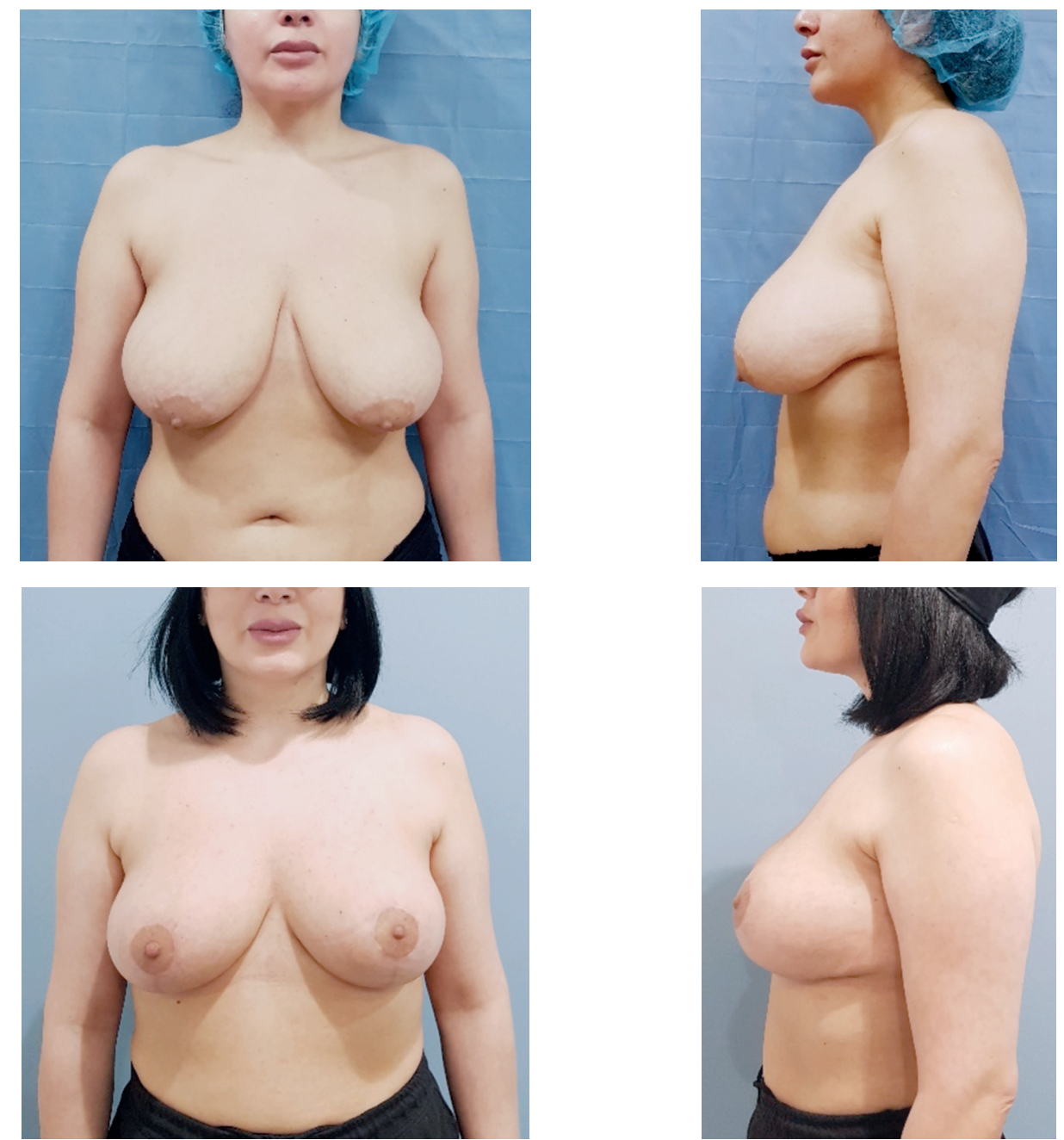

Fig. (3): Frontal and lateral views of pre-operative and 1 year post-operative follow-up of 43 years old patient.

Table (1): Post-operative complications.

\begin{tabular}{lll}
\hline Complications & $\begin{array}{c}\text { No. of } \\
\text { Breasts=74 }\end{array}$ & Percentage \\
\hline Hematoma & 1 & 1.3 \\
$\begin{array}{l}\text { Wound Dehiscence: } \\
\quad \text { Partial }\end{array}$ & 3 & \\
$\quad$ Complete & 0 & 4.1 \\
NAC Necrosis: & & 0 \\
$\quad$ Partial & 2 & \\
$\quad$ Complete & 0 & 2.7 \\
NAC reduced sensation: & & 0 \\
$\quad$ Transient & 11 & 11.8 \\
$\quad$ Permanent & 2 & 2.7 \\
Complete loss of sensation & 0 & 0 \\
Poor scarring & 14 & 18.9 \\
Fat necrosis & 2 & 2.7 \\
No complications & 39 & 52.7 \\
\hline
\end{tabular}

Table (2): Esthetic outcome evaluated by patients.

\begin{tabular}{lll}
\hline & $\begin{array}{c}\text { No. of } \\
\text { Patients }\end{array}$ & Percentage \\
& & \\
\hline Contour: & 2 & 5.5 \\
$\quad$ Not accepted & 9 & 24.3 \\
Accepted & 26 & 70.2 \\
Very pleased & & \\
Projection: & 5 & 13.5 \\
Accepted & 32 & 86.5 \\
Very pleased & & \\
Scar: & 1 & 2.7 \\
Very disappointed & 6 & 16.2 \\
Not accepted & 14 & 37.8 \\
Accepted & 16 & 43.2 \\
Very pleased & & \\
Symmetry: & 13 & 35.1 \\
Accepted & 24 & 64.9 \\
Very pleased & & \\
Aesthetic result satisfaction: & 1 & 2.7 \\
$\quad$ Not accepted & 8 & 21.6 \\
Accepted & & 75.7 \\
$\quad$ Very pleased & 28 & \\
\hline
\end{tabular}




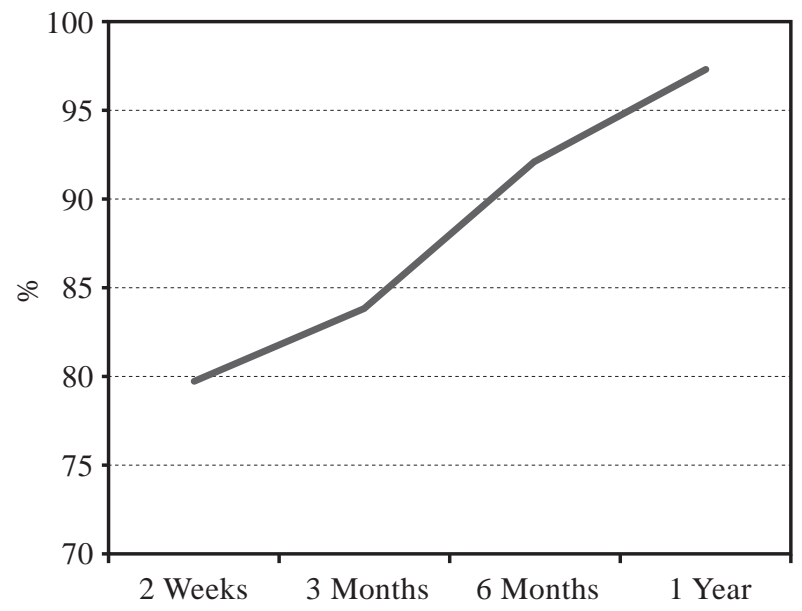

Graph (1): Shows percentage patients regained NAC sensation over time.

\section{DISCUSSION}

Coriddi (2013), in his study about patient satisfaction and well-being after reduction mammoplasty found that, satisfaction with breast appearance, physical, psychosocial and sexual well-being improved significantly despite the amount of excised weight. Isaias (2016), in his study to assess the effect of breast reduction on ability to work and productivity of women, it was found that it doesn't only improve women well-being but also, improved work capability and productivity in patients with breast hypertrophy in Brazil $[\mathbf{5 , 6 ]}$.

Two goals are the aim to be achieved by any of the described techniques for reduction mammoplasty: (1) To improve health problems associated with breasts hypertrophy (2) To obtain smaller size, youthful and aesthetically pleasing breast [7].

In this study the technique we used depended on supporting the superior pedicle with central glandular pedicle to avoid post-operative high incidence of NAC sensation loss or reduction that happens in superior pedicle reduction mammoplasty technique and ensure profuse blood supply to nipple areola complex by preserving most of internal thoracic artery perforators that emerges out through intercostal spaces just medial to breast meridian which in turn reduces possibility of NAC necrosis while, obtaining more projection, full upper pole of the breasts and getting long lasting good aesthetic outcome. The overall complication percentage excluding poor scarring was $14.8 \%$. We had no cases of total Nipple areola complex loss, low incidence of Nipple areola complex persistent reduced sensation after 1 year of followup $(2.7 \%)$ and no complete loss of sensation in any patient. Aesthetic outcome accepted by $97.3 \%$ of patients.

Schlenz I. et al., 2005 in his prospective study on nipple areola sensation condition after reduction mammoplasty using different techniques, by analyzing data of 23 patients done using superior pedicle reduction technique (Lassus, 10 patients; Lejour, 13 patients), mean resection weight in

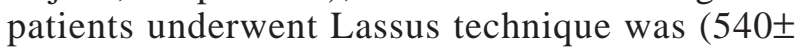
$123 \mathrm{~g}$ ) and those who underwent Lejour technique was $(390 \pm 112 \mathrm{~g})$. Three weeks postoperatively $47.8 \%$ of patient found to have nipple areola reduced sensation which was bilateral in $72.7 \%$ of patients and unilateral in $27.3 \%$ of patients. However; NAC sensation improved in most of patients, $13 \%$ of patients remained having NAC sensation loss [8].

Nadeau et al. (2015) in his study on 62 patients and Klinger et al. (2020) in his retrospective study on 823 patients who underwent superior pedicle reduction mammoplasty with preservation of central mound to support the superior pedicle, mean excised weight was $406 \mathrm{~g}$ and $860 \mathrm{~g}$ respectively. Postoperative complications in Nadeau et al. (2015) study was about $11.3 \%$. He had 1 case with hematoma, one case of cone deformity, 3 cases of infection, 8 cases with wound dehiscence and one patient developed unilateral Nipple areola complex necrosis. Klinger et al. (2020) noted that he had $35 \%$ overall complication rate, as he had 17 patients ( $2 \%)$ with seroma, 4 cases $(0.48 \%)$ with hematoma, 8 cases $(0.97 \%)$ with infection, 42 patients $(5.1 \%)$ with wound dehiscence, 25 patients $(5.1 \%)$ with partial nipple areola complex necrosis and 4 cases $(0.48 \%)$ with complete nipple areola complex necrosis. Transient loss of NAC sensation happened in 247 cases $(30 \%)$ and permanent loss of nipple areola complex sensation in 6 patients $(0.72 \%)$ $[9,10]$.

\section{Conclusion:}

Superior pedicle reduction mammoplasty supported by central glandular pedicle is simple, safe technique in breast reduction and provides advantages of: Ensuring profuse blood supply to nipple areola complex, reduces possibility of postoperative nipple areola complex reduction or loss of sensation, Gives very pleasant aesthetic outcome with good projection and long lasting results. Limited amount of tissue resection makes this surgical technique is not suitable for severely large breasts and doesn't replace superior pedicle reduction mammoplasty which allows larger volume of excised tissues in sever breast hypertrophy reduction. 


\section{REFERENCES}

1- Patricio Andrades and Arturo Prado. Understanding modern breast reduction techniques with a simplified approach. Journal of Plastic, Reconstructive \& Aesthetic Surgery, 61: 1284-1293, 2008.

2- Spear S.L. and Howard M.A.: Evolution of the vertical reduction mammaplasty. Plast. Reconstr. Surg., 112: 855$868,2003$.

3- Lista F. and Ahmad J.: Vertical scar reduction mammaplasty: 15-year experience including a review of 250 consecutive cases. Plast. Reconstr. Surg., 117 (7): 2152$2169,2006$.

4- Greuse M., Hamdi M. and DeMey A.: Breast sensitivity after vertical mammoplasty. Plast. Reconstr. Surg., 107: 970-976, 2001.

5- Coriddi M., Nadeau M., Taghizadeh M. and Taylor A.: Analysis of satisfaction and well-being following breast reduction using a validated survey instrument: The BREASTQ. Plast. Reconstr. Surg., 132: 285-290, 2013.
6- Isaias Vieira Cabral, et al.: Increased Capacity for Work and Productivity after Breast Reduction. Aesth. Surg. J., 36: 1-6, 2016.

7- Stevens W.G., Repta R., Pacella S.J., et al.: Safe and consistent outcomes of successfully combining breast surgery and abdominoplasty: An update. Aesth. Surg. J., 29 (2): 129-134, 2009.

8- Schlenz I., Rigel S., Schemper M., et al.: Alteration of nipple and areola sensitivity by reduction mammaplasty: A prospective comparison of five techniques. Plast. Reconstr. Surg., 115: 743-751, 2005.

9- Nadeau M., Gould D., Macias L., Spring M. and Stevens W.: Superior Pedicle Technique of Reduction Mammaplasty: A Stepwise Approach Aesthetic Surgery Journal, Vol. 35 (1): 94-104, 2015.

10- Klinger M., Klinger F., Maione L., Lisa A., Battistini A., Giannasi S., Veronesi A., Bandi V., Catania B., Barbera F., Lozito A., Caviggioli F. and Vinci V.: Superior Pedicle Breast Reduction with Prefiguration of Final Shape: A 10 -year Retrospective Study. Plast. Reconstr. Surg. Glob Open, 8: e3242, 2020. 